\title{
Morphologie des poils de Chiroptères d'Europe occidentale par étude au microscope électronique à balayage
}

par

\section{Yves TUPINIER}

Avec 4 planches, 1 photo dans le texte et 1 tableau.

Depuis un siècle et demi, on sait que les Chiroptères portent des poils dont la morphologie leur est particulière. Les études portant sur la faune européenne étant assez dispersées dans le temps comme dans l'espace, nous avons repris ce thème en faisant appel à la technique récente dans ce domaine, de la microscopie électronique à balayage.

\section{HISTORIQUE}

L'histoire de l'étude de la structure microscopique des poils de Chiroptères remonte à la première moitié du XIX ${ }^{\mathrm{e}}$ siècle quand BREWSTER en 1837 donne dans son Treatise on the microscope, la première description connue d'un poil de chauve-souris. Une note plus classique de J. QueKETT, lue en 1841 devant la Microscopical Society à Londres, donne des résultats d'observations portant sur seize espèces. Un bon nombre des structures actuellement connues sont ainsi présentées. Les différences dans la répartition des écailles le long du poil sont mises en évidence. On regrettera que cette recherche, orientée sur les possibilités que le microscope peut offrir au naturaliste, ne fasse pas mention du nom des espèces examinées.

Au milieu du XIX ${ }^{\mathrm{e}}$ siècle, F. KolenATI, travaillant sur la faune européenne, donne en 1857 ses observations d'où il ressort qu'une variation apparait d'une espèce à l'autre. De plus, la répartition des écailles le long de la tige du poil ainsi 
que la morphologie générale de ce dernier sont clairement décrites. En outre dans cette même étude, l'hypothèse que cette "structure intéressante des poils de Chiroptères " joue un rôle important dans la régulation thermique est nettement avancée. Tous ces résultats seront du reste repris par M. EISENTRAut dans Die deutschen Fledermäuse, en 1937. En 1859 dans sa Monographie, Kolenati donne pour chacune des espèces européennes les caractères spécifiques se fondant essentiellement sur le nombre des écailles qu'il considère comme coronales.

COOKE publie en 1868 des résultats obtenus sur des espèces exotiques. Une longue introduction fait le point des travaux antérieurs d'où il ressort que les travaux donnant des résultats d'observations de poils sont en bon nombre et que pour la plupart ce sont des études de microscopie pure, les espèces ayant donné matière à ces observations n'ayant qu'un intérêt secondaire et très lointain. La publication de MARCHI, que nous reprendrons en détail plus loin, marque la seconde moitié du XIX ${ }^{\mathrm{e}}$ siècle.

TOLDT, en 1907, fait figurer la structure du pelage, en reprenant les observations antérieures à ce sujet, dans les organes ou facteurs favorisant le vol. Un autre problème est soulevé en 1924 par Cole, celui de l'intérêt taxonomique offert par les différentes structures observées, tandis qu'à la même époque HAUSMAN précise une terminologie pour la description des écailles qui sera retenue par les auteurs modernes. Dans cette même décade, nous noterons une thèse soutenue par SPERLICH à Brno, sur les poils de Myotis myotis.

Les travaux plus récents, dont certains seront repris en détail dans les prochains paragraphes, portent sur une amélioration des connaissances des structures (HAUSMAN; NASON; BenEDICT). Parallèlement, les tentatives de mises sur pied de clés de détermination des poils se développent (MATHIAK; MAYER; BENEDICT). On note également des travaux sur la croissance du pelage pendant les toutes premières périodes de la vie des chauves-souris (KLIMA et GAISLER), ou la variation saisonnière (DWYER).

\section{STRUCTURE GÉNÉRALE DES POILS DE CHIROPTÈRES}

Depuis les travaux de Kolenati, la structure en fuseau des poils est bien connue par les publications, entre autres, de MARCHI, NASON, BENEDICT.

A partir de la base du poil, on trouve après le bulbe, une partie très courte sans écailles, sur une longueur de dix à vingt fois le diamètre. Les écailles apparaissent ensuite, d'abord très espacées les unes des autres leur densité en nombre augmente peu à peu. Elles s'imbriquent de plus en plus les unes dans les autres tandis que le diamètre du poil croît, sa valeur maximale étant atteinte au niveau du second tiers. Le dernier quart voit le poil s'effiler progressivement tandis que la densité des écailles diminue. 
Les poils des espèces européennes ne présentent pas de zone médullaire bien marquée. Le cortex, qui constitue la tige du poil, porte les écailles. Pour leur description j'ai repris la terminologie de HAUSMAN en l'adaptant à la langue française. Les écailles sont alors définies par les critères suivants:

a. forme générale: coronale si les écailles entourent complètement la tige du poil, imbriquée dans le cas contraire et souvent dans cette structure elles se chevauchent latéralement.

b. convergence avec trois possibilités: aprimée si le corps de l'écaille est parallèle à la tige du poil, divergente si un angle apparait, en général de 10 à $20^{\circ}$, entre le corps de l'écaille et l'axe du poil à cet endroit, de plus l'écaille reste en contact avec le cortex, divariquée, enfin, si l'angle de divergence croît, il est supérieur à $25^{\circ}$. Dans cette structure, le bord libre et environ le tiers supérieur du corps de l'écaille sont indépendants du cortex.

c. symétrie qui se définit soit par l'angle formé par le bord libre de l'écaille et l'axe du poil - si cette valeur est proche de $90^{\circ}$ l'écaille sera dite symétrique, sinon, la valeur de l'angle pouvant décroître jusqu'à $20^{\circ}$, elle sera dite alors asymétrique - soit par l'angle formé par l'axe du poil à cet endroit et l'axe principal. Dans ce cas l'asymétrie croît avec la valeur de l'angle.

d. dessin du bord libre. Ici les possibilités de variations sont plus grandes. J'ai retenu comme qualificatifs: entier pour un bord rectiligne sans accident, sinué si l'on observe des ondulations irrégulières pouvant être anguleuses, émarginé si le bord forme un angle rentrant bien marqué, denticulé si de petites dents régulières nombreuses, plus de quinze pour une demi-circonférence, sont présentes et denté si ce nombre est inférieur à dix, festonné pour une forme dentée où le bord, au lieu d'être constitué par une ligne brisée régulière, est formé par des demi-cercles en creux, enfin érodé pour traduire des bords libres très irréguliers visiblement altérés.

En général, c'est dans la zone moyenne, que KolEnati appelait le «tiers caractéristique » que les écailles présentent leur structure dans toutes les variétés tandis que dans la partie de plus grand diamètre l'uniformité est plutôt la règle.

\section{FONCTION DU PELAGE DES CHIROPTÈRES}

Le rôle joué par ces structures a, dès les premières études, attiré l'attention des chercheurs. KolENATI reconnait un moyen de régulation thermique, ToldT les relie au vol. RUDALL note que le contrôle de transfert d'eau est possible. Tout récemment D. J. Howell travaillant sur des Glossophaginae admet une adaptation au régime alimentaire. Pour ma part je suis assez enclin à penser qu'il s'agit d'une 
adaptation connexe du vol. Ces structures offrent la possibilité d'assurer le maximum d'effet de régulation thermique ou de transfert d'eau pour un poids minimal de matière. Cependant aucune de ces hypothèses n'explique la forme en massue des poils.

\section{DESCRIPTION DES POILS DES ESPÈCES EUROPÉENNES}

Dans sa «Monographie der europäischen Chiropteren », F. A. KolenatI donne pour chaque espèce citée une description des poils dont l'essentiel reste la longueur moyenne et surtout le nombre des écailles ainsi que leur répartition le long de la tige mais ne reprend pas les dessins publiés deux ans plus tôt, bien qu'ayant montré qu'il exitait des différences de morphologie.

Avec P. MARCHI, en 1873, on aborde une étude portant sur des espèces réparties dans l'ordre entier. Parmi les Chiroptères européens figurent dix-neuf espèces. Pour chacune d'elles il y a une courte description des poils ainsi que leur diamètre. La forme en fuseau est reconnue. Pour six de ces espèces des dessins complètent le texte, illustrant la variété de structure le long d'un même poil et selon les espèces. Cette étude restera pendant près d'un siècle en honneur et sera reprise dans des ouvrages comme le Traité de Zoologie, dans le chapitre consacré aux Chiroptères, en 1955 ou dans le chapitre homologue de Fauna d'Italia, rédigé par B. LANZA et publié en 1959.

De 1873 à nos jours très peu d'études ont porté sur la faune européenne. MANBY, en 1933, publie un cliché relatif à Miniopterus schreibersi. En 1950, dans son ouvrage Letucie mysi, A. P. KUZJAKIN donne des dessins des poils de 6 espèces selon des travaux datant de 1947 dus à N. S. Volshina qui, en 1951, à côté de quelques espèces exotiques décrit Rhinolophus hipposideros et $R$. mehelyi.

F. A. BENEDICT, en 1957, dans son ouvrage Hair structure as a generic character in bats, décrit les poils de sept espèces européennes dans le cadre de son étude par genre pour l'ensemble de l'ordre des Chiroptères.

En 1971, J. Gaisler, à l'occasion de ses travaux sur le développement du pelage en fonction de l'âge des Chauves-souris, donne une description pour quatre espèces.

\section{VALEUR TAXINOMIQUE}

Parallèlement à ces descriptions l'idée que cette morphologie particulière du pelage des Chauves-souris peut présenter une valeur taxinomique se développe. C'est ainsi que après Hausman en 1924, Cole qui étudie des Chiroptères des Philippines émet l'hypothèse que la structure des poils peut présenter un intérêt dans ce dessein. Après avoir examiné une vingtaine d'espèces, il conclut en écrivant 
Tableau récapitulatif des espèces européennes décrites

\begin{tabular}{|c|c|c|c|c|c|c|c|c|c|c|}
\hline & 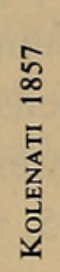 & 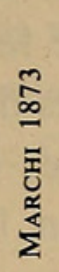 & 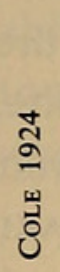 & 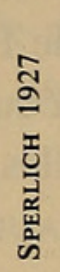 & 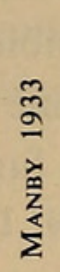 & 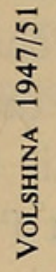 & 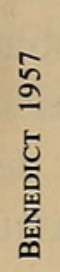 & 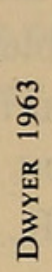 & 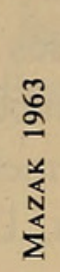 & 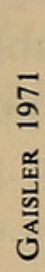 \\
\hline Rhinolophus ferrumequinum & 0 & $x$ & & & & & $x$ & & & \\
\hline Rhinolophus hipposideros & 0 & 0 & & & & 0 & & & & $x$ \\
\hline Rhinolophus blasii & 0 & $x$ & & & & & & & & \\
\hline Rhinolophus mehelyi & & & & & & 0 & & & & \\
\hline Tadarida teniotis & & 0 & & & & 0 & & & & \\
\hline $\begin{array}{l}\text { Plecotus auritus } \\
\text { Plecotus austriacus }\end{array}$ & 0 & $x$ & & & & & $x$ & & & \\
\hline Miniopterus schreibersi & 0 & 0 & & & 0 & & $x$ & $x$ & & $x$ \\
\hline $\begin{array}{l}\text { Barbastella barbastellus } \\
\text { Nyctalus lasiopterus }\end{array}$ & 0 & $x$ & & & & & & & & $x$ \\
\hline Nyctalus noctula & 0 & $x$ & & & & & & & & \\
\hline Nyctalus leisleri & & & & & & & & & & \\
\hline Myotis myotis & 0 & $x$ & & 0 & & 0 & $x$ & & $x$ & \\
\hline Myotis oxygnathus & & & & & & 0 & & & & \\
\hline Myotis dasycneme & 0 & 0 & & & & 0 & & & & \\
\hline Myotis capaccini & & & & & & & & & & \\
\hline Myotis daubentoni & 0 & $x$ & & & & & & & & \\
\hline Myotis mystacinus & 0 & $x$ & & & & 0 & $x$ & & & \\
\hline Myotis bechsteini & & $x$ & & & & & & & & \\
\hline Myotis nattereri & 0 & $x$ & & & & & & & & \\
\hline Myotis emarginatus & 0 & & & & & 0 & & & & $x$ \\
\hline Vespertilio murinus & 0 & $x$ & & & & & $x$ & & & \\
\hline Eptesicus nilssoni & 0 & & & & & & & & & \\
\hline Eptesicus serotinus & 0 & 0 & & & & & & & & \\
\hline Pipistrellus savii & 0 & $x$ & & & & & & & & \\
\hline Pipistrellus kuh!i & 0 & $x$ & & & & & & & & \\
\hline Pipistrellus pipistrellus & 0 & 0 & & & & & & & & \\
\hline Pipistrellus nathusii & 0 & & $x$ & & & & 0 & & & \\
\hline
\end{tabular}

que ce caractère n'apporte aucune aide pour distinguer deux espèces mais qu'il pourrait être intéressant au niveau du genre.

Par contre en 1938, MATHIAK publie des clés de détermination pour les poils des Mammifères du Michigan du Sud. Dix ans plus tard, NASON travaillant sur des espèces nord-américaines, décrit avec précision la variation de la structure pour un même poil qu'il divise lui aussi en quatre parties: la base, la zone moyenne, la zone de plus grand diamètre et l'extrémité en dégageant les caractères propres à chacune d'elles indépendamment de l'espèce et arrive aux mêmes conclusions que Cole sur la valeur taxinomique. 
Avec Volshina (1951) l'intérêt en faveur de la valeur pour la taxinomie revient et se développe avec W. V. MAYER qui publie en 1952 des clés de détermination pour l'ensemble des Mammifères de Californie retenant comme critère la longueur, le diamètre des poils, la répartition des pigments. BENEDICT ensuite, établit ses tables pour l'ensemble de l'ordre se fondant sur la forme des écailles, la présence ou l'absence de zone médullaire, les dimensions, et trouve des critères qui permettent de définir les familles avant les genres qui sont l'aboutissement des clés. D. R. Patil et P. N. Chaudhari, en 1965, reconnaissent une certaine valeur taxonomique mais émettent des réserves pour une signification phylogénétique en examinant des espèces sud-asiatiques. La même année, W. B. Miles travaillant sur la faune américaine montre qu'il existe une différence marquée entre les familles des Vespertilionidae et des Molossidae, en tenant compte des variations selon les parties proximale, centrale et distale des poils.

\section{MÉTHODE}

Jusqu'à ce jour le microscope photonique a été le principal instrument pour l'étude de la structure des poils. Cet instrument est surtout utilisé dans des examens par transparence, ce qui permet l'étude de la zone centrale ainsi que de la répartition des pigments. Par contre, dans l'observation des écailles, celles-ci apparaissent là où le maximum de matière est rassemblé sur le trajet de la lumière, tandis que là où l'épaisseur est minimale le tracé des écailles devient difficile à observer et peut échapper à l'observateur. C'est ainsi que par exemple dans les travaux anciens le poil de Tadarida teniotis présente des écailles en aiguilles latérales. Pour pallier cet inconvénient, certains auteurs comme MANBY, dès 1933, puis BENEDICT ont proposé pour les poils de Chiroptères une méthode d'observation indirecte consistant à substituer au poil sa propre empreinte. Toutefois ces méthodes délicates à mettre en œuvre n'ont pas eu de grands développements.

Pour notre étude, nous avons fait appel à la microscopie électronique à balayage, technique d'observation des surfaces qui offre l'avantage sur la microscopie photonique, de proposer une profondeur de champ 300 fois plus grande, qualité qui autorise une meilleure connaissance du relief et l'obtention de clichés photographiques de meilleure qualité. L'appareillage utilisé est du Type Stéréoscan de Cambridge E.I.L.

Pour permettre des comparaisons entre espèces, les prélèvements ont été effectués sur le dos de l'animal adulte au niveau des premières vertèbres dorsales, bien que la variation sur un même animal ne soit pas significative. De plus, pour toutes les espèces examinées les échantillons sont conservés en alcool. L'observation à l'aide du microscope électronique se faisant sous vide, après métallisation, nous avons craint que la deshydratation que cette contrainte impose, modifie 
la forme des écailles. Un contrôle avec un microscope photonique ne mit pas en évidence d'altérations significatives.

Les espèces examinées sont celles qui sont citées par MiLler à l'exception de Eptesicus sodalis. Par contre Plecotus austriacus (Fischer, 1827), non reconnue comme espèce valide en 1912 et réhabilitée depuis, a été ajoutée à la liste.

Une bonne partie des chauves-souris étudiées appartient aux collections du Muséum d'Histoire naturelle de Genève.

\section{Genre Rhinolophus}

\section{R. ferrumequinum (Schreber, 1774)}

La Jaudonnière (Vendée) 29 sept. 1968; collection Y. T. 81

La Balme les Grottes (Isère), 18 oct. 1968; collection Y. T. 85

R. hipposideros (Bechstein, 1800)

Arbigneu (Ain), 3 juillet 1966; collection Y. T. 53

R. blasii Peters, 1866

Tlemcem (Algérie); collection V. Aellen 626

R. euryale Blasius, 1853

La Balme les Grottes (Isère), fév. 1971; collection Y. T. 139

R. mehelyi Matschie, 1901

Obregon (Santander, Espagne), juillet 1964; collection Y. T. 19

L'examen des poils des espèces du genre Rhinolophus montre que les différentes espèces offrent une structure des écailles très voisine. On notera toutefois que le diamètre maximal peut atteindre $15 \mu \mathrm{m}$ pour $R$. ferrumequinum alors qu'il n'est que de $7-10 \mu \mathrm{m}$ pour les autres espèces.

Les écailles sont du type imbriqué, quelle que soit la partie du poil observée. Dans les mêmes conditions le caractère aprimé et asymétrique se maintient. A partir de l'origine du poil on rencontre tout d'abord de grandes écailles dont la longueur peut atteindre plus de deux fois le diamètre du poil. Leur asymétrie est très marquée donnant à l'ensemble l'aspect d'une ligne brisée dont les segments sont de taille assez constante mais qui tendent à se raccourcir lorsque l'on s'approche de la zone moyenne, leur longueur tend alors vers une valeur égale au diamètre puis dans la zone de diamètre maximal le nombre d'écailles pour une longueur équivalente à la valeur du diamètre, croît au dessus de un. Vers l'extrémité, le diamètre décroît et la longueur relative des écailles croît jusqu'à dépasser la valeur du diamètre.

Le bord libre des écailles, toujours bien en contact avec la tige du poil, se présente comme peu régulier et légèrement sinué. 


\section{Genre Tadarida}

\section{T. teniotis (Rafinesque, 1814)}

Villebois (Ain), 16 mars 1965; collection Y. T. 36

Ecailles de type coronal sur toute la longueur du poil, très divariquées à l'origine, puisque le rapport du diamètre maximal sur le diamètre minimal atteint deux, et très isolées les unes des autres, leur hauteur est supérieure au diamètre maximal. Pour cette région du poil, un examen au microscope photonique laisse apparaître de petites pointes latérales, le bord des écailles transparentes ne laissant qu'à peine entrevoir la structure coronale. Les écailles se resserrent peu à peu jusqu'au deuxième tiers de la longueur du poil. On compte alors, en moyenne, trois écailles pour une longueur égale au diamètre. Près de l'extrémité, les écailles s'écartent à nouveau, leur hauteur égale alors le diamètre. Ce dernier varie assez peu le long du poil et présente une valeur moyenne de $15 \mu \mathrm{m}$.

Le bord libre des écailles est denté, très régulier de l'origine à la moitié de la longueur du poil, puis, on observe des formes plus érodées là où la densité des écailles est maximale. Enfin vers l'extrémité, la hauteur des dentelures augmente, jusqu'à environ deux fois la longueur de la base, tandis qu'à l'origine hauteur et largeur sont égales.

\section{Genre Myotis}

M. myotis (Borkhausen, 1797)

Obregon (Santander, Espagne), 11 sept. 1967; collection Y. T. 73

M. oxygnathus (Monticelli, 1885)

Crémieu (Isère), 23 juin 1969; collection Y. T. 103

M. dasycneme (Boie, 1825)

Soisson (Aisne), 7 nov. 1959; collection V. Aellen 2445

M. daubentoni (Leisler in Kuhl, 1819)

Détain (Côte d'Or), sept. 1954; collection Y. T. 7

M. capaccinii (Bonaparte, 1837)

Grotte du Queire (Ariège), août 1951; collection Muséum de Genève 968-2

M. mystacinus (Leisler in Kuhl, 1819)

Nuits-Saint-Georges (Côte d'Or), avril 1955; collection Y. T. 11

Saint-Pierre d'Entremont (Savoie), janvier 1971; collection Y. T. 155

M. bechsteini (Leisler in Kuhl, 1818)

Saint-Rambert en Bugey (Ain), 8 janv. 1966; collection Y. T. 39

Curtil-Vergy (Côte d'Or), 11 avril 1966; collection T. Y. 48 
M. nattereri (Kuhl, 1818)

Claveisolles (Rhône), mars 1970; collection Y. T. 116

M. emarginatus (E. Géoffroy, 1806)

Détain (Côte d’Or), avril 1966; collection Y. T. 51

Les neuf espèces du genre Myotis qui ont été examinées présentent une morphologie très voisine. Si les écailles sont imbriquées et aprimées sur toute la longueur du poil, leur symétrie est très variable dans les mêmes conditions. C'est ainsi que près de la base, dans la région moyenne et l'extrémité, c'est le caractère asymétrique qui domine, tandis que dans la zone de plus grand diamètre, la symétrie tend à devenir la règle. Dans les parties proximales et distales, une symétrie peut apparaître sur quelques écailles qui se suivent. Près de l'origine les écailles sont longues, jusqu'à trois fois leur diamètre, l'asymétrie domine et l'on retrouve sur quelques segments un aspect de ligne brisée .On remarque toutefois, des écailles imbriquées symétriques qui peuvent présenter un aspect divariqué très marqué. Dans la zone moyenne, les écailles perdent peu à peu leur caractère divergent et deviennent de plus en plus imbriquées. On observe des zones où les bords libres sont parallèles. Dans la partie de plus grand diamètre - $16 \mu \mathrm{m}$ contre 13 dans la zone moyenne et 6 à la base pour un même poil —, les écailles augmentent en densité le long de la tige, leur hauteur peut descendre en dessous du diamètre. Près de l'extrémité l'aspect déjà vu dans la zone moyenne se retrouve.

Le bord libre des écailles est en général entier à la base et dans la partie moyenne et devient sinué ou émarginé dans la zone de plus grand diamètre.

\section{Genre Barbastella}

\section{B. barbastellus (Schreber, 1774)}

Claveisolles (Rhône), déc. 1968; collection Y. T. 88

Les poils ne présentent que des écailles imbriquées en toutes zones, en général très asymétriques sauf dans la partie de plus grand diamètre. Celles de la base, bien que relativement longues, ne dépassent guère deux fois leur diamètre minimal et cela, tout près de l'origine (10 à 15 écailles). La densité augmente rapidement le caractère asymétrique s'estompant légèrement. Dans la zone moyenne on note des parties où les écailles imbriquées ont leur bord libre parallèle faisant un angle de $40-45^{\circ}$ avec l'axe du poil. La zone de plus grand diamètre voit la densité augmenter. On compte alors deux à trois écailles pour une longueur égale au diamètre. Le poil prend un aspect général cylindrique et compact qui se maintient jusqu'à l'extrémité. Les bords libres entiers ne sont sinués que dans la zone de plus grand diamètre et à l'extrémité. 


\section{Genre Plecotus}

\section{P. auritus (Linné, 1758)}

Claveisolles (Rhône), déc. 1968; collection Y. T. 91

Claveisolles (Rhône), déc. 1969; collection Y. T. 112

P. austriacus (Fischer, 1827)

Crémieux (Isère), jan. 1966; collection Y. T. 41

Lantigné (Rhône), déc. 1970; collection Y. T. 146

La structure présentée par ces deux espèces apparaît comme très voisine. Les écailles sont imbriquées. Toutefois, on note que leur caractère asymétrique, qui reste la règle générale, peut s'atténuer dans la moitié proximale jusqu'à présenter un aspect coronal qui peut persister sur quelques écailles voisines. Ce détail est plus fréquent chez $P$. austriacus. A la base du poil, les écailles sont de grande taille puisque leur longueur peut atteindre trois fois leur diamètre qui se situe vers $4 \mu \mathrm{m}$ dans les parties les plus frêles. L'asymétrie des écailles, bien que très marquée, ne donne pas l'aspect d'une ligne brisée. Dans la partie moyenne le diamètre varie de 7 à $14 \mu \mathrm{m}$. Les écailles sont alors très divariquées tout en restant en contact avec le cortex. C'est dans cette zone que les écailles d'aspect coronal se rencontrent. En suivant le poil, le caractère divariqué s'atténue et disparait complètement dans la zone de diamètre maximal dont la valeur se situe vers $14 \mu \mathrm{m}$. Le poil est presque cylindrique. Les écailles très aprimées ont une longueur égale à la moitié de la valeur du diamètre. Vers l'extrémité distale les écailles redeviennent relativement plus grandes alors que le diamètre décroît rapidement.

Le bord libre des écailles, toujours entier, assez régulier dans la première moitié, s'altère dans la zone de plus grand diamètre où il peut être sinué ou même émarginé.

\section{Genres Eptesicus et Vespertilio}

\section{E. serotinus (Schreber, 1774)}

Genève, sept. 1968; collection Muséum de Genève 3325

E. nilssoni (Keyserling et Blasius, 1839)

Jura bernois, février 1951; collection Muséum de Genève 390

V. murinus Linné, 1758

Col de Brétolet (Valais), sept. 1963; collection Muséum de Genève 2747

Ces deux genres très proches l'un de l'autre ne présentent pas de différences significatives. C'est ainsi que pour les trois espèces, nous avons près de l'origine des écailles longues, asymétriques, divergentes - le diamètre du poil varie de 4 à $7 \mu \mathrm{m}$ de la base au sommet des écailles. Celles-ci dans la partie moyenne 
se rapprochent les unes des autres, leur hauteur est alors voisine du diamètre. La divergence s'atténue ainsi que l'asymétrie, ce qui donne un aspect coronal qui s'amplifie dans la zone de diamètre maximal $(10 \mu \mathrm{m})$. Près de l'extrémité, on retrouve l'aspect de la zone moyenne. Le bord libre, entier, devient rapidement émarginé. Dans la partie moyenne la profondeur de l'échancrure est maximale.

\section{Genre Nyctalus}

N. lasiopterus (Schreber, 1780)

Col de Cou (Valais), oct. 1962; collection Muséum de Genève 2683

N. noctula (Schreber, 1774)

Nyon (Vaud), oct. 1969; collection Muséum de Genève 3350

N. leisleri (Kuhl, 1818)

Col de Brétolet (Valais), août 1964; collection Muséum de Genève

A son origine, le poil des Noctules présente des écailles divergentes peu asymétriques. La divergence est bien marquée surtout chez $N$. leisleri ( 7 et $13 \mu \mathrm{m}$ pour les diamètres minimal et maximal). Dans la partie moyenne, les écailles imbriquées et aprimées peuvent, par leur disposition, présenter une symétrie qui leur donne un aspect coronal. Dans la zone de plus grand diamètre $(12$ à $13 \mu \mathrm{m})$, le caractère fusiforme est ici moins marqué. Cette structure des écailles persiste, avec toutefois une augmentation de la densité, le long de la tige. Leur hauteur ne descend que peu au dessous de la valeur du diamètre.

Dans les trois espèces, le bord libre est sinué et même émarginé.

\section{Genre Pipistrellus}

P. savii (Bonaparte, 1837)

Gênes (Italie), août 1969; collection Muséum de Genève 669

P. kuhli (Natterer in Kuhl, 1819)

Lyon, nov. 1952; collection Y. T. 3

Lyon, fév. 1956; collection Y. T. 10

Chalamont (Ain), déc. 1968; collection Y. T. 93

P. pipistrellus (Schreber, 1774)

Dombes (Ain), mars 1967; collection Y. T. 114

P. nathusii (Keyserling et Blasius, 1839)

Allamond (Ain), mars 1967; collection Y. T. 56

Ce genre est le seul de la faune européenne à présenter des variations significatives selon les espèces. Comme caractères communs, il faut noter que les écailles sont imbriquées et que souvent deux peuvent être associées dans une 
structure coronale. Près de la base et dans la partie moyenne la divergence est très marquée puisque l'on peut relever chez $P$. savii 4 et $12 \mu \mathrm{m}$ comm valeur minimale et maximale du diamètre et, chez $P$. kuhli 5 et 13 . Dans cette même zone, les écailles fortement divariquées ne sont en contact avec le cortex qu'à leur base.

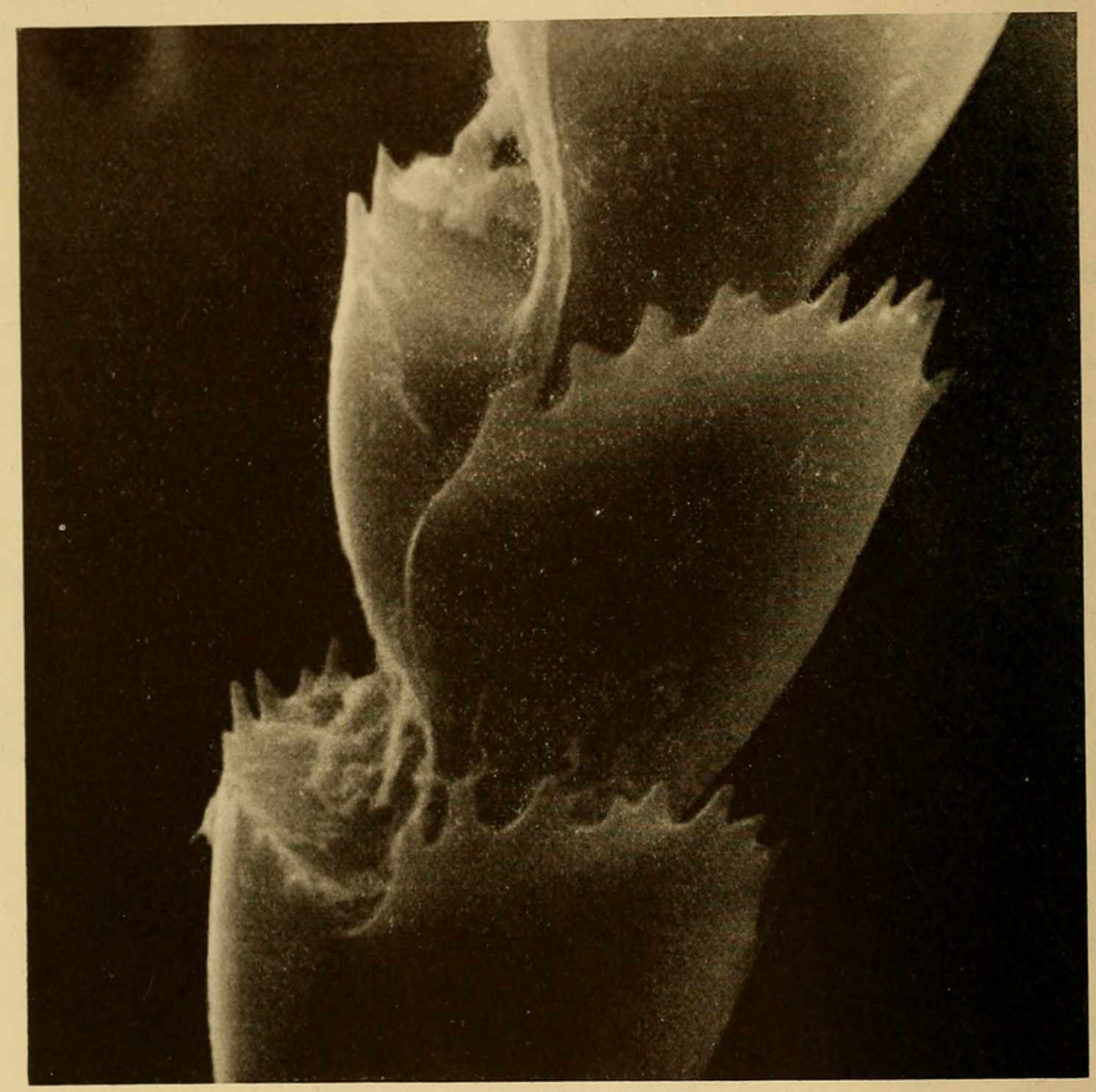

FIG. 48.

Poil de Pipistrellus kuhli dans la partie caractéristique, où les écailles associées prennent un aspect coronal (grossissement 5000).

Dans la zone dite de plus grand diamètre, les écailles deviennent plus nombreuses (deux pour une longueur de tige égale au diamètre), plus aprimées. La forme générale du poil devient cylindrique. A l'extrémité les écailles se séparent et l'on retrouve les caractères de la zone moyenne.

Le bord libre des écailles présente également des variations spécifiques, surtout près de l'origine et dans la partie moyenne. C'est ainsi que chez $P$. kuhli on observe de fines dentelures qui alternent avec des courbes en creux, alors que 
P. nathusii présente un bord libre en dents de scie. Les deux autres espèces ont une limite régulière. Par contre dans la zone de diamètre maximal et à l'extrémité du poil, on observe chez ces quatre espèces des écailles dont le bord est érodé et l'on peut reconnaître des structures qui peuvent ressembler aux dentelures telles que l'on peut les voir chez $P$. nathusii dans sa zone moyenne. L'état de fraicheur permet seul de reconnaître cette dernière espèce. Ces détails n'apparaissent pas toujours dans un examen à l'aide d'un microscope photonique, car vus par transparence les bords libres ne sont visibles que latéralement. Cette technique laisse donc voir une structure coronale, les bords latéraux des écailles ne pouvant être mis en évidence.

\section{Genre Miniopterus}

M. schreibersi (Natterer in Kuhl, 1819)

Obregon (Santander, Espagne), juillet 1964; collection Y. T. 31

Crémieux (Isère), jan. 1966; collection Y. T. 43

Sur toute la longueur du poil, les écailles sont imbriquées, très asymétriques, surtout dans la moitié proximale et toujours aprimées. A partir de la base, on observe une zone d'écailles très longues, donnant au poil l'aspect d'une ligne brisée dont les segments atteignent $15 \mu \mathrm{m}$ pour un diamètre de $3 \mu \mathrm{m}$. Dans la partie moyenne les segments deviennent plus courts, $10 \mu \mathrm{m}$, alors que le diamètre atteint $6 \mu \mathrm{m}$. Dans la zone de diamètre maximal, $8 \mu \mathrm{m}$, l'aspect général tend vers une structure cylindrique, les écailles ayant une longueur légèrement supérieure au diamètre. Vers l'extrémité distale les écailles apparaissent divergentes. Le bord libre entier et régulier, présente quelques fines dentelures à la base du poil.

\section{CONCLUSIONS}

Ces observations ont confirmé un certain nombre de points bien connus par les travaux antérieurs comme la forme en massue ou la répartition des écailles le long du poil. Il n'en a pas été de même dans la structure de ces dernières. C'est ainsi que dans beaucoup de cas les formes coronales au sens strict, c'est à dire qu'une seule écaille fasse le tour complet de la tige du poil, sont en fait des associations dont l'ensemble laisse croire que cette structure existe, les bords latéraux étant difficilement observables par les méthodes de microscopie photonique. Comme seuls exemples selon cette définition nous n'aurons plus que Tadarida teniotis et Pipistrellus nathusii. Dans tous les autres cas nous sommes en présence du type imbriqué.

Ces travaux ont montré que seule la zone moyenne, la " partie caractéristique » de Kolenati, présente une variété de structure alors que dans la région de dia- 
mètre maximal les formes rencontrées sont loin d'être propres aux Chiroptères. Si l'on se limite à cette région typique du poil, il ressort que certains types sont plus fréquents. C'est le cas de ceux qui présentent des écailles d'aspect coronal entières, aprimées, au bord libre sinueux. Ils ont été vus chez un nombre élevé d'espèces: Rhinolophus ferrumequinum, Plecotus auritus, Miniopterus schreibersi, Myotis myotis, M. oxygnathus, M. bechsteini, Eptesicus serotinus, E. nilssoni... C'est également le cas de ceux dont les écailles sont entières, divergentes, irrégulièrement asymétriques que nous avons notées chez Rhinolophus hipposideros, $R$. mehelyi, Plecotus austriacus, Miniopterus schreibersi, Myotis myotis, M.oxygnathus, $M$. dasycneme, $M$. daubentoni, $M$. mystacinus, $M$. nattereri, M. emarginatus... Par contre, on trouve des poils caractéristiques seulement de deux espèces: Tadarida teniotis et Pipistrellus nathusii dont le bord libre, serratiforme, d'écailles divergentes ou divariquées, est très typique. Le cas où le caractère spécifique est le plus marqué, est celui de Pipistrellus kuhli, seule espèce dont les écailles sont festonnées en creux.

Nous avons porté une attention particulière sur le cas d'espèces dont la morphologie est très semblable, comme Myotis myotis et $M$. oxygnathus ou Plecotus auritus et $P$. austriacus. Les différences sont trop peu accusées pour être retenues comme critère spécifique. On ne retrouve pas, à propos de cette morphologie pileuse, de différences aussi nettes que par exemple, dans l'examen des baculums.

Quant à l'intérêt pour la taxinomie, nous retiendrons que seules quelques espèces peuvent être reconnues par leurs poils, et que dans la majorité des cas ici présents la variabilité des formes est telle qu'il est hasardeux de vouloir retenir des critères de détermination.

\section{RÉSUMÉ}

Ces observations, utilisant les techniques de la microscopie électronique à balayage, ont confirmé les descriptions antérieures sur la structure générale des poils. Par contre, il a été montré que dans de nombreux cas connus d'écailles coronales, on est en présence d'écailles imbriquées associées. La valeur taxinomique n'est à retenir avec intérêt que pour quelques espèces bien définies (Tadarida teniotis, Pipistrellus nathusii, P. kuhli, P. pipistrellus). En outre, des descriptions et illustrations originales sont données pour Rhinolophus euryale, Plecotus austriacus, Nyctalus lasiopterus, N. leisleri, Myotis capaccini.

\section{ZUSAMMENFASSUNG}

Diese Beobachtungen, die die Abtastungstechnik im Elektronmikroskop gebrauchten, haben die bisherigen Beschreibungen über den Aufbau der Haare bestätigt. Dagegen hat man gezeigt dass in vielen bekannten Fällen von kranz- 
artigen Schuppen, es sich um dachziegelförmigen assozierte Schuppen handelt. Der taxonomische Wert ist nur für einige ganz bestimmte Arten von Interesse (Tadarida teniotis, Pipistrellus nathusii, P. kuhli, P. pipistrellus). Originalbeschreibungen und Illustrationen sind ausserdem für Rhinolophus euryale, Plecotus austriacus, Nyctalus lasiopterus, N. leisleri und Myotis capaccini angegeben.

\section{SUMMARY}

These observations, made using the techniques of scanning electron microscopy have substantiated previous descriptions of the general structure of the hair. On the other hand, it has been demonstrated that in many known instances of coronal scales these are imbricate scales in association. The value to taxonomy is of interest only for certain well-defined species (Tadarida teniotis, Pipistrellus nathusii, $P$. kuhli, $P$. pipistrellus). In addition, original descriptions and illustrations are provided for Rhinolophus euryale, Plecotus austriacus, Nyctalus lasiopterus, N. leisleri, Myotis capaccini.

\section{BIBLIOGRAPHIE}

BENEDICT, F. A. 1957. Hair structure as a generic character in bats. University of California Press, Berkeley and Los Angeles.

Bieber, H. und U. SCHMIDT. 1971. Untersuchungen zur Haarfärbung bei Vampirfledermäusen (Desmodus rotundus murinus Wagner). Z. Säugetierk. 36: 280-283.

CARTer, B. C. and T. G. Dilworth. 1971. A simple technique for revealing the surface pattern of hair. Am. Midl. Nat. 85 (1): 260-262.

Cole, H. I. 1924. Taxonomic value of hair in Chiroptera. Philipp. J. Sci. 24: 117-120.

CоOKE, M. C. 1868 . On the hairs of Indian bats. J. Queckett microsc. Club 1: 33-45 et 55-63.

DANFORTH, C. H. 1925. Hair in its relation to questions of homology and phylogeny. Am. J. Anat. 36 (1): 47-68.

DWYER, P. D. 1963. Seasonal changes in pelage of Miniopterus schreibersi blepotis (Chiroptera) in North-Eastern New South Wales. Aust. J. Zool. 11: 290-300.

Eisentraut, M. 1937. Die deutschen Fledermäuse. Schöps, Leipzig.

Forster, A. 1926-27. Contribution à l'étude du peaucier chez les Cheiroptères, son adaptation au vol. Archs Anat. Hist. Embryol. 6: 347-359.

GaISLER, J. 1971. Vergleichende Studie über das Haarkleid der Fledertiere (Chiroptera). Prirodov. Pr. Cesk. Akad. Ved 8: 1-44.

Grassé, P. P. 1955. In: Traité de Zoologie, tome XVII, fasc. 2, ordre des Chiroptères, pages 1729-1863. Ed. Masson, Paris.

Hausman, L. A. 1920. Structural characteristics of the hair of mammals. Am. Nat. 54 (635): 496-523. 
Hausman, L. A. 1924. Further studies of the relationships of the structural characters of mammalian hair. Am. Nat. 58: 544-557.

Hausman, L. A. 1930. Recent studies of hair structure relationships. Scient. Mon., N. Y. 30: 258-277.

Hausman, L. A. 1932. The cortical fusi of mammalian hair shafts. Am. Nat. 66: 461-470.

Howell, D. J. 1972. Physiological adaptations of bat pollinators and their hosts. 3e Congrès intern. de Recherches sur les Chauves-souris, Plitvice, Yougoslavie, septembre 1972, résumés, page 41.

Howell, D. J. and N. HodgKins. 1971. Indications of adaptive significancy in bat hairs shown by Stereoscan photography. The Second Southwestern Symposium on Bat Research, nov. 1971, University of New-Mexico, U.S.A., résumés.

Klima, M. and J. Gaisler, 1967. Study on growth juvenile pelage in bats. I. Vespertilionidae. II. Rhinolophidae, Hipposideridae. Zool. Listy 16: 111-124 et 343-354.

Kolenati, F. 1857. Beiträge zur Naturgeschichte der europäischen Chiroptern. Allg. dt. naturh. Ztg. Dresden-Leipzig 3: 1-50.

Kolenati, F. 1859. Monographie der europäischen Chiropteren. Ib. Mähr.-Schles. Ges. Ackerb. Brünn: 1-156.

Kuzjakin, A. P. 1950. Letucie mysi. Sovetskaja Nauka, Moscou.

Lanza, B. 1959. In: Fauna d'Italia, Mammalia. Chitoptera. Ed. Cálderini Bologna.

Mahrle, G., R. Christenhusz und C. Orfanos. 1969. Haar und Haarcuticula im Raster-Elektronenmikroskop. Arch. klin. exp. Derm. 295: 295-300.

Manby, J. 1933. Scale structure of the hair of the bat. Nature, Lond. 132: 244-245.

Marchi, P. 1873. Sulla morphologia dei peli nei Chirotteri. Atti Soc. ital. Sci. nat. 15 (5): 513-541.

MathiaK, H. A. 1938. A key to hairs of the mammals of Southern Michigan. J. Wildl. Mgmt 2 (4): 251-268.

MAYER, W. V. 1952. The hairs of California mammals with keys to the dorsal guard hairs of California mammals. Am. Midl. Nat. 48 (2): 480-512.

MAZAK, V. 1963. Hair growth in large mouse-eared bat, Myotis myotis myotis (Borkh. 1797) Mammalia, Chiroptera during its prenatal and early postnatal life. Věst. čsl. Spol. zool. 27 (3): 234-242.

Miles, W. B. 1965. Studies of the cuticula structure of the hairs of Kansas bats. Search, Univ. Kansas Publ. 5: 48-50.

MülleR, K. 1939. Über den Bau der koronalen Schüppchen der Säugetierhaares. Zool. Anz. 126 (5/6): 97-107.

Nason, E. S. 1948. Morphology of hair of Eastern North American bats. Am. Midl. Nat. 39 (2): 345-361.

Patil, D. and P. N. Chaudhari. 1965. Note on the hair of some bats. J. Bombay nat. Hist. Soc. 62 (3): 539-542.

QueKeTt, J. 1844. On the structure of bat's hair. Trans. microsc. Soc. Lond. 1: 58-62.

Rudall, K. M. 1941. The structure of the hair cuticule. Proc. Leeds phil. lit. Soc. 4 (1): 13-18.

Sperlich, F. 1927-28. Die Haare der Fledermaus: Vespertilio murinus. Diss. Vet. Fak. Brno. (non vu).

Toldt, K. 1907. Über die Hautgebilde der Chiropteren. Verh. zool. bot. Ges. Wien 57 : 83-91. 
TUPINIER, Y. 1972. Etude de la morphologie des poils des Chiroptères d'Europe occidentale au microscope électronique à balayage. 3e Congrès intern. de Recherches sur les Chauves-souris, Plitvice, Yougoslavie, sept. 1972, résumés, page 35.

Volshina, N. G. 1951. Variations du pelage des Chiroptères (en russe). Bull. Soc. Nat. Moscou, Biol. 56: 21-30.

WiLliams, C. S. 1938. Aids to the identification of mole and shrew hairs with general comments on hair structure and hair determination. J. Wildl. Mgmt 2 (4): 239-250.

Adresse de l'auteur

Laboratoire de Biologie souterraine

Département de Biologie animale et Zoologie

Université Claude Bernard Lyon I

43, Boulevard du 11 novembre 1918

F-69-Villeurbanne

France. 


\section{PLANCHE I}

- la pointe des poils est orientée vers le haut.

— grossissement 2000 (à plus ou moins $5 \%$ près).

1 - Rhinolophus euryale

2 - " "

$3-\quad " \quad$

$4-\infty \quad 》$

5 - $\quad$ "blasii

6 - » mehelyi

7 - $\gg$ hipposideros

8 - $\quad$ ferrumequinum

9 - Miniopterus schreibersi

10 - »

11 - 》

12 \ 》 origine

partie moyenne

partie de diamètre maximal

extrémité

partie moyenne

partie moyenne

partie moyenne

partie de diamètre maximal

origine

partie moyenne

partie de diamètre maximal extrémité

\section{PLANCHE II}

- la pointe des poils est orientée vers le haut.

- grossissement 2000 (à plus ou moins $5 \%$ près).

13 - Myotis myotis

14 - " oxygnathus

15 - " dasycneme

16 - " capaccinii

17 - $\gg$ nattereri

18 - " "

19 - " bechsteini

20 - " "

21 - " daubentoni

22 - " emarginatus

23 - " mystacinus

24 - 》 》 partie de diamètre maximal partie de diamètre maximal partie de diamètre maximal origine

partie moyenne

extrémité

partie moyenne

partie de diamètre maximal partie de diamètre maximal partie de diamètre maximal origine

partie moyenne 


\section{PLANCHE III}

- la pointe des poils est orientée vers le haut.

- grossissement 2000 (à plus ou moins 5\% près).

\section{5 - Plecotus auritus}

26 - » »

$27-\gg \quad »$

$28-\gg \gg$

29 - $\gg$ austriacus

30 - » »

$31-» \quad »$

$32-» \quad »$

33 - Eptesicus serotinus

34 - " nilssoni

35 - Vespertilio murinus

36 - » origine

partie moyenne

partie de diamètre maximal extrémité

origine

origine

partie moyenne

partie de diamètre maximal partie moyenne partie moyenne

origine

partie moyenne

\section{PLANCHE IV}

- la pointe des poils est orientée vers le haut.

- grossissement 2000 (à plus ou moins 5\% près).

37 - Pipistrellus savii

38 - $\gg$ pipistrellus

39 - $\gg \quad k u h l i$

40 - " "

41 - \ nathusii

42 - Barbastella barbastellus

43 - Nyctalus lasiopterus

44 - $\gg$ noctula

45 - » leisleri

46 - » »

47 - Tadarida teniotis origine

partie moyenne

partie moyenne

partie de diamètre maximal

partie moyenne

partie moyenne

partie moyenne

partie moyenne

origine

partie moyenne

partie moyenne 


\section{$2 \mathrm{BHL}$ Biodiversity Heritage Library}

Tupinier, Yves. 1973. "Morphologie des poils de Chiroptères d'Europe occidentale par étude au microscope électronique à balayage." Revue suisse de zoologie 80, 635-653. https://doi.org/10.5962/bhl.part.75960.

View This Item Online: https://www.biodiversitylibrary.org/item/126816

DOI: https://doi.org/10.5962/bhl.part.75960

Permalink: https://www.biodiversitylibrary.org/partpdf/75960

\section{Holding Institution}

Smithsonian Libraries

\section{Sponsored by}

Biodiversity Heritage Library

\section{Copyright \& Reuse}

Copyright Status: In Copyright. Digitized with the permission of the rights holder.

Rights Holder: Muséum d'histoire naturelle - Ville de Genève License: http://creativecommons.org/licenses/by-nc-sa/3.0/

Rights: https://www.biodiversitylibrary.org/permissions/

This document was created from content at the Biodiversity Heritage Library, the world's largest open access digital library for biodiversity literature and archives. Visit BHL at https://www.biodiversitylibrary.org. 